\section{Tone injection-based cancellation technique for nonlinear distortion reduction of modulated signals in BAW resonators}

\author{
Marta González-Rodríguez (i) । \\ Carlos Collado I \\ José M. González-Arbesú （） ～Jordi Mateu | \\ Gabriel Montoro | Pere Lluis Gilabert
}

Components and Systems for Communications (CSC) Group, Department of Signal Theory and Communications (TSC), Universitat Politècnica de Catalunya (UPC) BarcelonaTech, Barcelona, Spain

\section{Correspondence}

Marta González-Rodríguez, Components and Systems for Communications (CSC) Group, Department of Signal Theory and Communications (TSC), Universitat Politècnica de Catalunya (UPC) BarcelonaTech c/ Jordi Girona, 31, (08034) Barcelona, Spain.

Email: marta.gonzalez.rodriguez@tsc.upc.edu

\section{Funding information}

Secretary of Universities and Research of the Generalitat de Catalunya and the European Social Fund, Grant/Award Number: FI_B-00136; Spanish Government, Grant/Award Numbers: 2017 SGR-813,

TEC2017-84817-C2-2-R, TEC2017-88343-C4-2-R

\begin{abstract}
A technique for the reduction of third-order intermodulation distortion (IMD3) occurring in bulk acoustic wave (BAW) filters and multiplexers is presented. The method consists of using the intrinsic second-order intermodulation distortion generated by BAW filters and multiplexers themselves by injecting an externally controlled amplitude and phase modulated signal at low frequency to compensate for the IMD3. The proposed technique has been experimentally verified by performing IMD3 measurements in a single BAW resonator subject to several modulated signals under a nonlinear scenario.
\end{abstract}

\section{KE YWORDS}

BAW, cancellation system, distortion, intermodulation, nonlinearities

\section{1 | INTRODUCTION}

Through its bulk acoustic wave (BAW) configuration, acoustic technology has become one of the most important technologies for the development of RF and microwave filters in handset devices that gives response to the current and forthcoming stringent bandwidth scenarios demanded by the wireless communications industry. Despite its superior performance in terms of signal rejection, insertion losses, and degree of miniaturization, its intrinsic nonlinear behavior can compromise the overall system's linearity and thus quality performance. ${ }^{1}$ In particular, second-order nonlinearities that generate second-order intermodulation distortion (IMD2) or second harmonics (H2) have received the focus of the scientific community in recent years since these are the predominant nonlinear effects in BAW devices, and successful techniques have been developed to mitigate them.

Current scenarios with spectral efficient multicarrier waveforms and technologies, such as carrier aggregation and multiple input multiple output, together with the coexistence of concurrent multi-band or multiple Radio Access Technology communications, open up new challenges in the field of nonlinear characterization and mitigation. Third-order manifestations of the nonlinear effects, such as third-order intermodulation distortion (IMD3), might also have an impact on the system's performance, reducing the receiver's sensitivity. This reawakens the interests on the full understanding and modeling of such nonlinear effects ${ }^{2}$ and even more important, on seeking methods to mitigate the IMD3 phenomenon occurring in complex filtering architectures. ${ }^{3}$

All-digital cancellation techniques have been previously used to mitigate potential receiver desensitization due to intermodulation products ${ }^{4}$ generated by the transmitter leakage. In Reference 5, we already applied the tone injection technique to cancel out the passive IMD3 generated in a BAW resonator. However, going from the concept to a real and practical application in a modern communication transceiver is obviously a long way and, whereas the proposed cancellation technique in Reference 5 was validated using single-tone fundamental sources, in this article we go a step further toward to a practical implementation, by proving that this technique can be applied to modulated signals. The proposed architecture allows for IMD3 suppression by using the intrinsic IMD2 generated in the device itself (not in the measurement setup), when a phase and amplitude-controlled injected modulated signal mixes-up with one of the two modulated fundamental signals. 
The key factor of this proposal is that the technique exploits the fact that the generation of second-order nonlinearities is higher than the generation of third-order intermodulation products in BAW devices. ${ }^{6}$ This means that a generated low frequency and low power canceling signal may produce an IMD2 signal that cancels out the IMD3 generated by the two high-power transmitting signals. Therefore, this technique is not suitable when second-order nonlinearities are low, such as in symmetric SAW resonators. $^{7}$

This article demonstrates, as a proof-of-concept, the validity of the proposed technique in a laboratory environment. The application of this technique to modulated signals is not straightforward and requires of a cancellation system that, to the best authors' knowledge, has not been previously reported in the literature.

The applicability of this technique in real communications systems is beyond the scope of this article. However, its applicability lays in the fact that the required canceling signal that must be generated is a low power and low frequency signal that can be digitally generated in the low frequency stages of the transceiver in a similar way as the baseband predistorted signals are generated when linearizing high-power amplifiers. ${ }^{8}$ Unlike the laboratory setup no component is required aside from the generation of the lowpower low-frequency signal at the same time than the data to be transmitted.

The article is organized as follows. Section 2 summarizes the nonlinear equations describing the nonlinear effects in electro-acoustic resonators. Moreover, a simplified theoretical cancellation demonstration is also outlined. Section 3 presents a brief description of the measurement system and provides details on implementation aspects, such as signal generation and instruments configuration. Section 4 describes the measurements of an on-wafer, BAW-type solidly mounted resonator (SMR) when fed with modulated signals (simple amplitude modulated signals have been used to prove the technique). Finally, conclusions are given in Section 5.

\section{2 | NONLINEAR EQUATIONS ANALYSIS}

The source of electroacoustic devices nonlinearities has been widely studied in the past using equivalent circuit models. Although the generation of those nonlinear effects can be diverse, it can be explained using well-known constitutive nonlinear equations. ${ }^{2}$

\section{1 | Constitutive nonlinear equations}

As previously reported in Reference 2, the constitutive nonlinear equations describe the relation between the stress
$(T)$ and strain $(S)$, and the electric displacement $(D)$ and field $(E)$ in the piezoelectric layer:

$$
\begin{aligned}
& T=c^{E} S-e S+\Delta T \\
& D=e S+\varepsilon^{S}+\Delta D
\end{aligned}
$$

where $e$ is the piezoelectric constant, $c^{E}$ the elastic constant, and $\varepsilon^{S}$ the dielectric constant. As seen in Reference 5, the nonlinear terms $\Delta T$ and $\Delta D$ are:

$$
\begin{aligned}
& \Delta T=c_{2}^{E} \frac{S^{2}}{2}+c_{3}^{E} \frac{S^{3}}{6}-\varphi_{3} \frac{E^{2}}{2}+\varphi_{5} S E \\
& \Delta D=\varepsilon_{2}^{E} \frac{E^{2}}{2}+\varepsilon_{3}^{E} \frac{E^{3}}{6}-\varphi_{5} \frac{S^{2}}{2}+\varphi_{3} S E
\end{aligned}
$$

which depend on several second-order $\left(c_{2}^{E}, \varphi_{3}, \varphi_{5}, \varepsilon_{2}^{E}\right)$ and third-order $\left(c_{3}^{E}, \varepsilon_{3}^{E}\right)$ derivative constants. These terms identify the nonlinear behavior of the piezoelectric layer. Since identifying which are the most relevant or dominant terms falls out of the main scope of this article, only $\varphi_{5}$ and $c_{3}^{E}$ nonlinear terms will be considered for the H2 and IMD3 generation in the next section, ${ }^{10}$ thus, the other nonlinear terms will be disregarded. Note that, from a practical point of view, the cancellation process is independent of the specific terms that may cause second- and third-order nonlinearities.

\subsection{IMD3 cancellation expressions}

Derivation of equations for the IMD and $\mathrm{H} 2$ applied to an ideal film bulk acoustic resonator (FBAR) were done in the past by the authors in References 9 and 10. Obtaining closed-form expressions for the nonlinearities in a SMR-type BAW resonator is not an easy task because, in addition to the piezoelectric material, there are several materials composing the resonator and some of them can generate nonlinearities. $^{10}$ In this subsection, a simplified theoretical demonstration of the cancellation is presented just to illustrate an example of the cancellation mechanism.

Let us assume that a device is fed with two high power transmitted signals with fundamental frequencies $f_{T x 1}$ and $f_{T x 2}$, and phasor voltages $V_{i n, T x 1}$ and $V_{i n, T x 2}$. If, we intend to cancel out the IMD3 that falls at $2 f_{T x 1}-f_{T x 2}$, the injected signal will have the frequency $f_{I T}=f_{T x 2}-f_{T x 1}$, and phasor voltage $V_{\text {in, IT. }}$. Following the same reasoning as in Reference 11, it is possible to obtain an expression for the IMD2 and the IMD3 considering only the most relevant second- and third-order derivative constants explained before. Assuming these hypotheses, IMD3 of the two fundamental signals can be caused by direct generation of third-order nonlinear constants $\left(c_{3}^{E}\right.$ in this example) or remix effects due to second-order nonlinear constants $\left(\varphi_{5}\right) .{ }^{11}$ Therefore, we can obtain the following voltage nonlinear expressions for the IMD2 and IMD3 that falls at the same frequency $2 f_{T x 1}-f_{T x 2}$, 


$$
\begin{aligned}
& V_{\text {out }, I M D 2}=K(\omega) \varphi_{5} V_{i n, T x 1} V_{i n, I T}^{*} \\
& V_{\text {out }, I M D 3}=\left(J(\omega) \varphi_{5}^{2}+H(\omega) c_{3}^{E}\right) V_{i n, T x 1}^{2} V_{i n, T x 2}^{*}
\end{aligned}
$$

where, $K(\omega), J(\omega)$, and $H(\omega)$ represent the frequency dependent response of the device at given frequencies and where * denotes complex conjugate. Matching the two equations and isolating $V_{\mathrm{in} \text {, IT }}$ we get:

$$
V_{i n, T T}=\left(\frac{J(\omega)}{K(\omega)} \varphi_{5}+\frac{H(\omega) c_{3}^{E}}{K(\omega) \varphi_{5}}\right)^{*} V_{i n, T x 1}^{*} V_{i n, T x 2}
$$

If only direct generation is considered, Equation (4) can be simplified just neglecting the first term inside the parenthesis. Instead, if we consider IMD3 generation due to remix effects, only the first term inside the parenthesis is considered. More nonlinear constants and different materials could be considered in the equations just adding more terms. Note that in practical situations no generality is lost since finally the phase and amplitude of the injected tone maybe experimentally adjusted.

\section{3 | MEASUREMENT SYSTEM}

Figure 1 outlines the proposed cancellation system, having at the left-top the two high-power (HP) signals $\left(f_{T x 1}\right.$ and $\left.f_{T x 2}\right)$ feeding the device under test (DUT) and at the right-bottom the injected signal at frequency $f_{I T}$ (used to generate the proper IMD2). Specifically, the injected signal, denoted as $f_{I T}$, was an injected baseband modulated signal having the same modulation than $f_{T x 2}$. The cancellation technique allows us to cancel the generated by the DUT IMD3 nonlinearities at both sides of the fundamental signals, $2 f_{T x 1}-f_{T x 2}$ and/or $2 f_{T x 2}-f_{T x 1}$. Previous to the cancellation procedure it is verified that the setup IMD3 levels without the DUT are below the noise floor of the measurement setup. Further details about the measurement system were described in. ${ }^{5}$

\section{1 | Signal generation}

Regarding the signals generation, a personal computer oversees the instrument control along with the code used for the generation of the modulated signals. In this experiment, $f_{T x I}$ signal is just a single-tone generated using a Phase Matrix FSW-0010 frequency synthesizer (FS), and $f_{T x 2}$ and $f_{I T}$ are both modulated signals generated using two Agilent N5182A MXG vector signal generators (VSG), being both VSG's and the FS synchronized through an Agilent N9020A MXG signal analyzer (SA). Moreover, since both signals must be modulated, first step is to initialize both VSGs setting their powers and frequencies and disabling the arbitrary waveform generation (ARB) capabilities.

Later, the digital to analog converter range of the VSG is set in such a way that a waveform file must be in the volatile memory of the dual ARB player. Following that, the sample clock rate for the dual ARB format is set and both the automatic leveling control is used to maintain the signal generator's output power level, as well as the ARB function is turned on.

Moreover, a MATLAB script generates a pair of inphase (I) and quadrature $(\mathrm{Q})$ vectors having the VSGs configured in such a way that they expect the input files of said pair of vectors, reading them, and generating the desired modulated signals.

Furthermore, as we are using two VSGs as baseband generators, system synchronization is needed. Moreover, a trigger is used to control the data transmission and deciding when the signal generator transmits the modulated signal. The trigger type of the master VSG corresponding to the $f_{T x 2}$

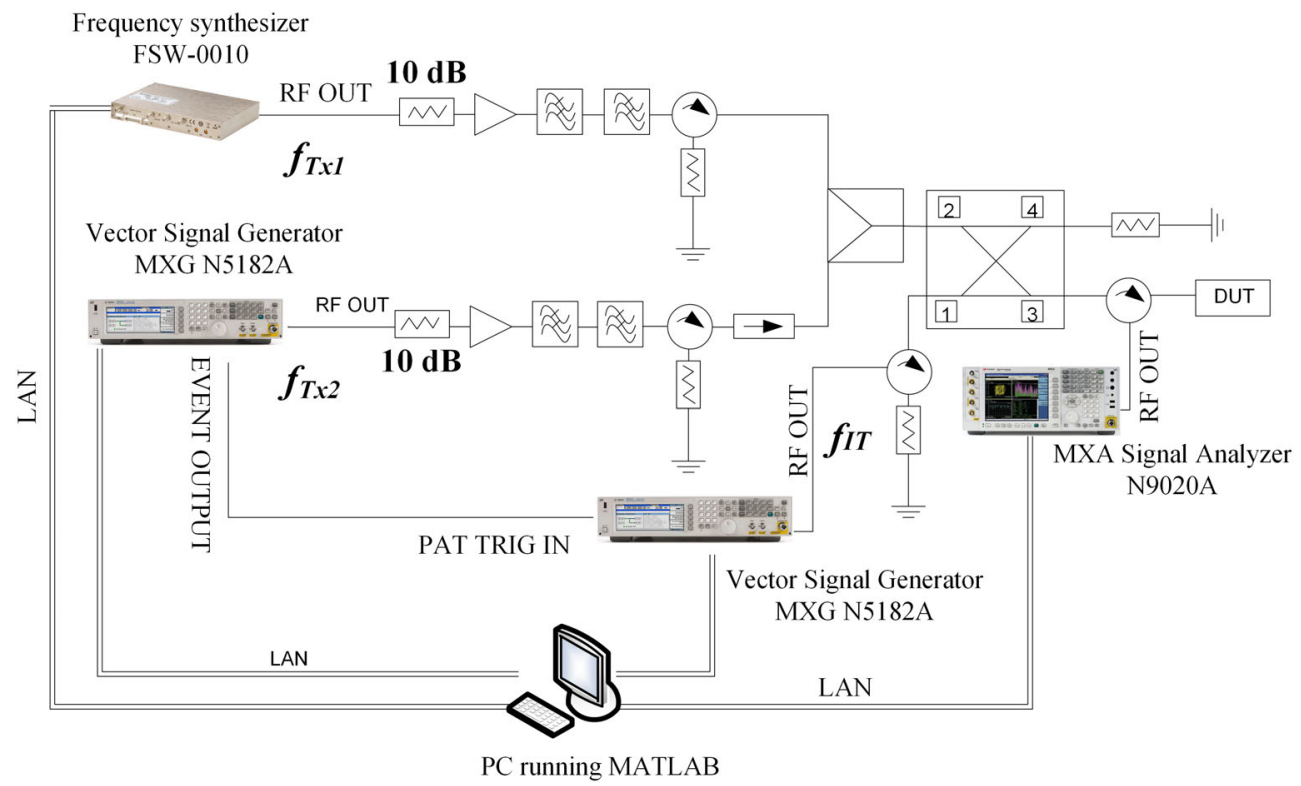

FIG URE 1 Tone-injection experimental cancellation setup block diagram [Color figure can be viewed at wileyonlinelibrary.com] 
signal is set to continuous and free run, and the trigger type of the slave VSG corresponding to the $f_{I T}$ signal is set to trigger and run. Moreover, the trigger source of the $f_{I T}$ signal is set to external, considering that plays the waveform after receiving master trigger. System synchronization occurs after the master signal generator sends an event pulse and propagates it to the slave by using the EVENT output signal to trigger the slave. Prior to this, the slave is configured to wait for this event pulse through the PAT TRIG connector. System delay is reduced to a minimum in both VSGs by setting the I/Q delay properly to compensate for any latency between the EVENT output signal and the RF output.

Apart from that, a proper synchronization between the VSGs carriers, the FS, and the SA using the REF IN and the $10 \mathrm{MHZ}$ OUT connectors is done.

\section{4 | EXPERIMENTAL RESULTS}

Measurements of an on-wafer resonator were done. The tested resonator is a commercial SMR BAW resonator based on a piezoelectric AlN layer sandwiched between two electrodes and lying on an acoustic reflector. Its series resonance frequency is $2.438 \mathrm{GHz}$ and its shunt resonance frequency is $2.502 \mathrm{GHz}$. The resonator area is $1.4 \cdot 10^{-8} \mathrm{~m}^{2}$.

Several nonlinear measurements were done to validate the application of the proposed technique to the cancellation of modulated signals. Specifically, two different case scenarios were established: an amplitude modulated (AM) signal and a random noise modulated signal. These modulations are taken as an example of signals that take-up a wide

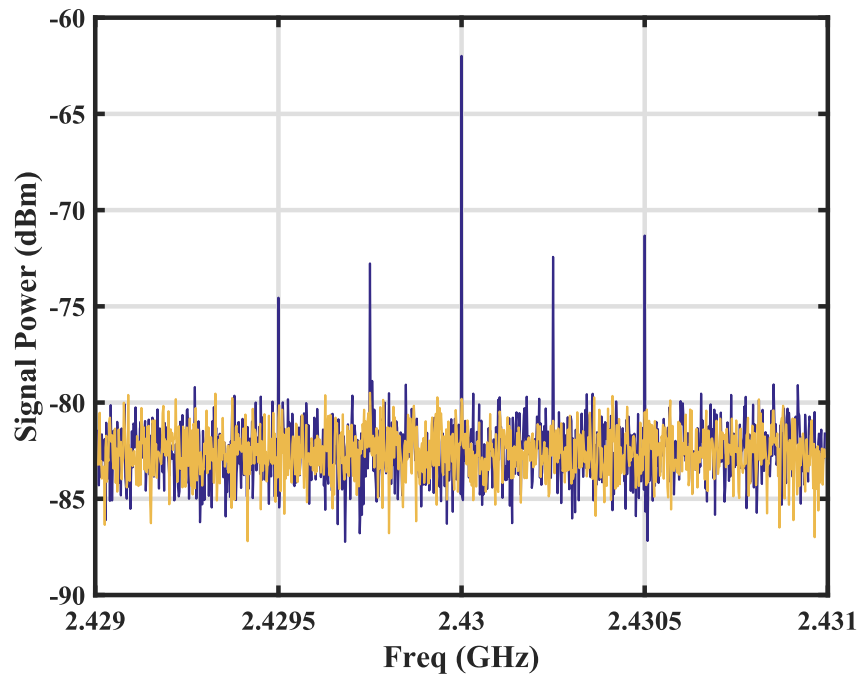

FIGURE 2 IMD3 cancellation example of a two-tone AM modulated signal with a $1 \mathrm{MHz}$ bandwidth and modulation index of 0.3 . The blue trace represents the response without cancellation and the yellow trace the canceled response. AM, amplitude modulated; IMD3, third-order intermodulation distortion [Color figure can be viewed at wileyonlinelibrary.com] spectrum while allowing to have a power spectral density superior to that of the noise level.

To speed up the cancellation process, and before activating the modulation of the carriers, some previous steps are done, as a sort of precalibration. First, the generated IMD3 (signal to be canceled) is measured feeding the DUT with only the two HP tones. Second, if we intend to cancel the IMD3 at $2 f_{T x 1}-f_{T x 2}$, the required injected tone is set on and the signal at $f_{T x 2}$ is set off, producing an IMD2 tone at the same frequency than the targeted IMD3. The magnitude of the injected tone is adjusted to provide the same level of IMD2 than the IMD3 measured in the first step.

Once this is done, all the modulated carriers are set on and the cancellation process starts by adjusting the phase of the injected signal and making minor magnitude corrections.

\section{1 | AM measurements}

In this example the fundamental tones $f_{T x 1}$ and $f_{T x 2}$ were located at 2.45 and $2.47 \mathrm{GHz}$ with input powers 22.6 and $21.0 \mathrm{dBm}$, respectively. The injected tone was placed at the same frequency of the separation between the fundamental tones $f_{I T}=20 \mathrm{MHz}$ and the required power of the injected tone was set to $-17.2 \mathrm{dBm}$ in the precalibration process. Then, the modulations are activated.

Figure 2 depicts the measured IMD3 at $2 f_{T x 1}-f_{T x 2}$, using a two-tone AM modulation for $f_{T x 2}$. The blue trace shows the IMD3 response before activating the cancellation algorithm. The injected tone at $f_{I T}$ is then modulated with the same baseband signal, and fine tuning its magnitude values and adjusting its phase, the IMD3 is canceled out (in yellow) with the own IMD2 generated by the resonator. Figure 2 shows almost $20 \mathrm{~dB}$ cancellation at the carrier frequency of an AM signal having $1 \mathrm{MHz}$ of spectral bandwidth. The actual cancellation level can be higher since the canceled level is below the noise floor of the spectrum analyzer.

\section{2 | Random noise modulation measurements}

In this second example the central frequency of the signals $f_{T x 1}$ and $f_{T x 2}$ were located at 2.45 and $2.55 \mathrm{GHz}$, respectively, and the injected signal at $f_{I T}=100 \mathrm{MHz}$. In this case, $f_{T x 1}$ is generated as a single-tone and $f_{T x 2}$ and $f_{I T}$ are carriers modulated with random noise in order to fully occupy the signal bandwidth. Two different bandwidths have been tested, $100 \mathrm{kHz}$ and $1 \mathrm{MHz}$, leaving the power of the injected canceling signal constant. Figure 3 depicts the measured third-order random noise IMD3 response at $2 f_{T x 1}-f_{T x 2}$. Following the same reasoning as in Section 4.1, the cancellation is shown in the whole bandwidth.

It is important to notice that the signal power level in this scenario was smaller in comparison with the AM modulation case, since the same available power must be distributed in a greater bandwidth given the nature of the noise-modulated 


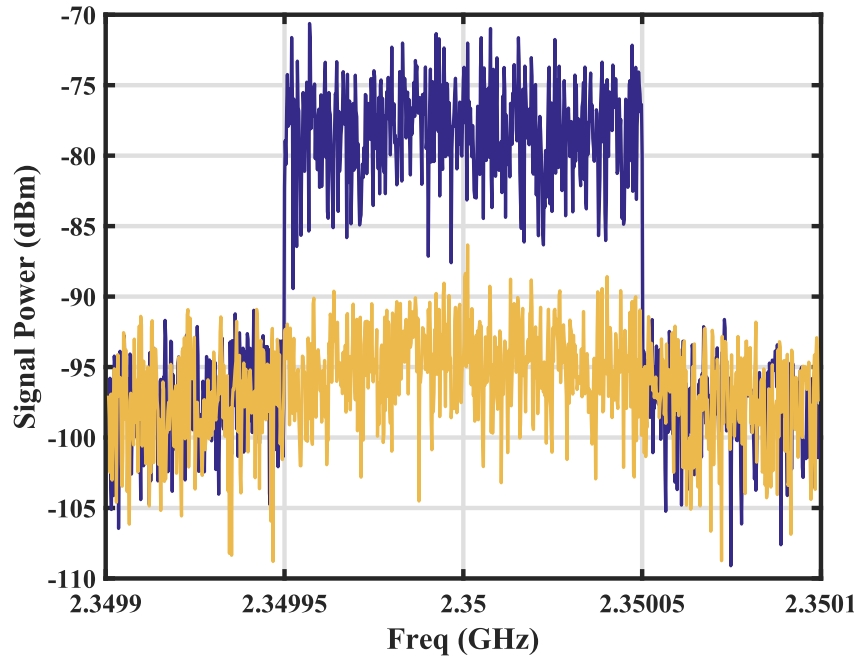

FIG URE 3 IMD3 cancellation example of a random noise modulated signal with a $100 \mathrm{kHz}$ bandwidth. The blue trace represents the response without cancellation and the yellow trace the canceled response. IMD3, third-order intermodulation distortion [Color figure can be viewed at wileyonlinelibrary.com]

signal. Consequently, signal power levels on Figures 3 and 4 are different in comparison with Figure 2.

Figure 3 shows cancellation figures between 15 and $20 \mathrm{~dB}$ in the entire bandwidth for the $100 \mathrm{kHz}$ case, and, at least, $10 \mathrm{~dB}$ for the $1 \mathrm{MHz}$ case in Figure 4.

\section{5 | CONCLUSIONS}

This article formulates and experimentally evaluates a suitable method for an active cancellation of the harmful IMD3 occurring in complex wireless scenarios where electroacoustic devices are used on the filtering stages. Validation of such concept requires of a careful development of a cancellation setup where a given modulated signal is used to feed the DUT at the operating frequency band and at the same time with a lower frequency version of the modulated signal, whose amplitude and phase can be controlled in real-time. Details of the measurement setup and the experiments are detailed in section 4 to demonstrate the suitability of the proposed technique. This concept can indeed be applied in more complex scenarios where several modulated signals coexist, and several electroacoustic devices are leading to several nonlinear manifestations. For example, in frequency selective devices like a quadplexer where the transmitter and receiver share the antenna, IMD3 generation can cause interfering signals at a given receiver band, affecting its sensitivity. The conceptual advantage of the application of this technique to real transceivers is that the low frequency canceling signals could be digitally generated into the same transceiver if the IMD3 to be canceled is coming from two HP transmitting signals, whose properties are well known by the own transceiver.

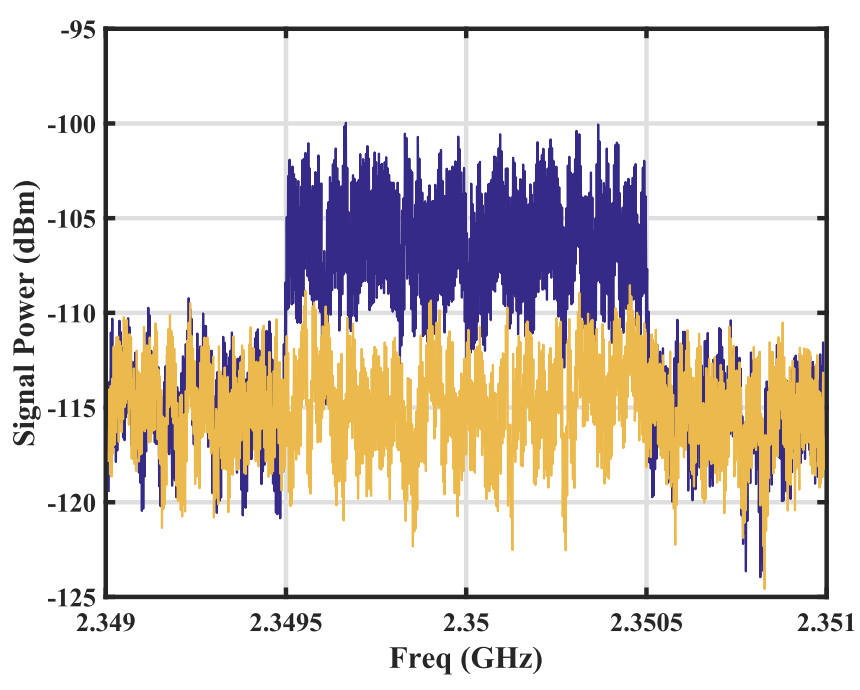

FIGURE 4 IMD3 cancellation example of a random noise modulated signal with $1 \mathrm{MHz}$ bandwidth. The blue trace represents the response without cancellation and the yellow trace the canceled response. IMD3, third-order intermodulation distortion [Color figure can be viewed at wileyonlinelibrary.com]

This technique in combination with real time adaptive algorithms, which act on the parameters that characterize the all-digital canceling signal, could even cancel the variation of the IMD3 signal due to the external environment. Changes of the input impedance of the duplexer antenna could modify the generated IMD3 to be canceled falling in the receiver frequency band, requiring therefore adaptive algorithms to cancel out this spurious signal.

\section{ACKNOWLEDGMENTS}

This work was supported in part by the Spanish Government through grants TEC2017-84817-C2-2-R, TEC2017-88343C4-2-R, and 2017 SGR-813. Research was also supported by the Secretary of Universities and Research of the Generalitat de Catalunya and the European Social Fund through grant FI_B-00136.

\section{OR CID}

Marta González-Rodríguez (D) https://orcid.org/0000-0001-79222674

José M. González-Arbesú (D) https://orcid.org/0000-00029601-0988

\section{REFERENCES}

[1] Hashimoto KY. RF Bulk Acoustic Wave Filters for Communications. Boston: Artech House; 2009.

[2] Shim DS, Feld DA. A general nonlinear mason model of arbitrary nonlinearities in piezoelectric film. Presented at Proceedings of the IEEE International Ultrasonics Symposium (IUS), San Diego, (USA), 2010, pp. 295-300. 
[3] Rocas E, Mateu J, Collado C, et al. Nonlinear performance of BAW filters including BST capacitors. Radioengineering. 2014;23(1):369.

[4] Dabag H-T, Gheidi H, Gudem P, Asbeck PM. All-digital cancellation technique to mitigate self-jamming in uplink carrier aggregation in cellular handsets. Presented at IEEE MTT-S International Microwave Symposium Digest (MTT), Seattle, Washington (USA), 2013, pp. 1-3.

[5] González-Rodríguez M, Collado C, González-Arbesú JM, Mateu J. New technique to cancel IMD3 in electroacoustic filters. Presented at IEEE International Ultrasonics Symposium (IUS), Kobe (Japan), 2018. pp. 1-4.

[6] Rocas E, Collado C, Mateu J, Orloff ND, Booth JC, Aigner R. Electro-thermo-mechanical model for bulk acoustic wave resonators. IEEE Trans Ultrason Ferroelectr Freq Control. 2013;60(11): 2389-2403.

[7] Nakagawa R, Kyoya H, Shimizu H, Kihara T. Effective suppression method for 2nd nonlinear signals of SAW devices. Presented at IEEE International Ultrasonics Symposium (IUS), Chicago, (USA), 2014, pp. 782-786.

[8] Cheng K-KM, Leung C-S. A novel generalized low-frequency signal-injection method for multistage amplifier linearization. IEEE Trans Microw Theory Tech. 2003;51(2):553-559.
[9] Rocas E, Collado C, Padilla A, Mateu J, O'Callaghan JM. Nonlinear distributed model for IMD prediction in BAW resonators. Presented at IEEE Ultrasonics Symposium (IUS), Beijing (China), 2008, pp.1557-1560.

[10] Collado C, Rocas E, Mateu J, Padilla A, O'Callaghan JM. Nonlinear distributed model for bulk acoustic wave resonators. IEEE Trans Microw Theory Tech. 2009;57(12):30193029.

[11] Collado C, Mateu J, Garcia-Pastor D, et al. Nonlinear effects of $\mathrm{SiO} 2$ layers in bulk acoustic wave resonators. IEEE Trans Microw Theory Tech. 2018;66(4):1773-1779.

\section{How to cite this article: González-Rodríguez M,} Collado C, González-Arbesú JM, Mateu J, Montoro G, Gilabert PL. Tone injection-based cancellation technique for nonlinear distortion reduction of modulated signals in BAW resonators. Microw Opt Technol Lett. 2020;1-6. https://doi.org/10.1002/mop. 32670 